\title{
A influência da intensidade emocional no reconhecimento de emoções em faces por crianças brasileiras
}

The importance of emotional intensity on the recognition of facial emotions by Brazilian children

\author{
DOI: $10.11144 /$ Javeriana.upsy15-5.iier \\ Recepção: 05 Agosto 2016 | Aprovação: 30 Novembro 2016
}

\author{
Juliana Silva Rocha Aguiar ${ }^{a}$ \\ Universidade de Brasília, Brasil \\ Ana Idalina de Paiva Silva \\ Universidade Federal de Goiás, Brasil \\ Carla Silva Rocha Aguiar \\ Universidade de Brasília, Brasil \\ Nelson Torro-Alves \\ Universidade Federal da Paraíba, Brasil \\ WÂnia Cristina de Souza \\ Universidade de Brasília, Brasil
}

\begin{tabular}{lcc}
\hline aCorresponding & author. & E-mail: \\
juliana.aguiar.psi@gmail.com &
\end{tabular}

Para citar este artículo: Rocha Aguiar, J. S., Silva, A. I., Rocha Aguiar, C. S., Torro-Alves, N., de Souza, W. C. (2016). A influência da intensidade emocional no reconhecimento de emoções em faces por crianças brasileiras. Universitas Psychologica, 15(5). http://dx.doi.org/10.11144/Javeriana.upsy15-5.iier

\section{RESUMO}

A capacidade de reconhecer emoções nos rostos é essencial para a interação humana e ocorre desde a infância. Hipótese: a pesquisa utilizando a técnica de morphing supõe que as crianças precisam de uma maior ou menor intensidade da expressão emocional para percebelas. Objetivo: Analisar o reconhecimento emocional facial na infância, utilizando uma tarefa com diferença na intensidade emocional. Método: Foi aplicado um teste de Reconhecimento de Emoções Faciais para Crianças a 28 crianças com idades entre 7 e 11 anos. $O$ teste apresentava 168 imagens de emoções básicas, manipuladas pelo morphing. Resultados: A idade se apresenta como uma tendência de crescimento da possibilidade de sucesso na tarefa; maior quantidade de respostas corretas para alegria e pior performance para o medo; e a intensidade emocional aumenta em $42 \%$ a probabilidade de sucesso por cada unidade de aumento na intensidade. Conclusão: Esses resultados são relevantes já que demostram que o reconhecimento emocional em diferentes níveis é um método mais sensível.

\section{Palavras-chave}

Reconhecimento de Emoção em Face; Desenvolvimento Infantil; Morphing; Intensidade Emocional.

\section{ABSTRACT}

The ability to recognize emotions in faces is essential to human interaction and occurs since childhood. Hypothesis: research using the morphing technique assume that children require greater or lesser intensity of emotional expression to perceive it. Objective: to examine the emotional recognition of faces in childhood, using a task with emotional intensity variation. Method: it was applied a Test of Facial Emotion Recognition for Children to 28 children between 7 and 11 years, of both sexes, which presented 168 faces manipulated by the morphing technique, of the six 
Juliana Silva Rocha Aguiar, Ana Idalina de Paiva Silva, Carla Silva Rocha Aguiar, Nelson Torro-Alves, ET AL.

basic emotions. Results: age as a trend growth of the likelihood of success at the task; more right answers for happiness and worst performances for fear; and the emotional intensity increasing at $42 \%$ the chance of success by every unit of intensity. Conclusion: these findings are relevant because they show the recognition of emotions at different levels as a more sensitive method.

Keywords

Facial Emotion Recognition; Child Development; Morphing; Emotional Intensity.

\section{Introdução}

A comunicação não-verbal é essencial à interação entre os seres humanos, possibilitando a transmissão de mensagens importantes à adequação comportamental e à sobrevivência (Meletti, 2016; Siegman \& Feldstein, 2014; Tanaka et al., 2012). Tais meios de comunicação possuem elementos inatos, sendo a face o principal artifício de expressão nas relações humanas (Darwin, 1872/2004).

A face é capaz de transmitir diversas informações, tais como aquelas relacionadas ao gênero, idade, identidade e emoção sentida pelo indivíduo (Bruce \& Young, 1986; Dhall, Goecke, Joshi, Wagner \& Gedeon, 2013; Hofmann, Suvak \& Litz, 2006; Murtaza, Sharif, Raza \& Shah, 2013). O giro fusiforme é uma das principais áreas responsáveis pelo reconhecimento da identidade facial (Kawasaki et al., 2011; Weiner \& Zilles, 2015), estabelecendo conexões com outras partes do encéfalo que atuam no sistema emocional (Gauthier, Tarr, Anderson, Skudlarski \& Gore, 1999; Haxby et al., 2000).

As emoções estão associadas à produção de respostas corporais que nos preparam para a ação (Rodrigues \& Rocha, 2016). As chamadas expressões das "emoções básicas" compreendem a alegria, tristeza, raiva, nojo, surpresa e medo e têm sido observadas em uma grande diversidade de culturas humanas (Ekman, 1992). A alegria é a expressão mais facilmente identificada em qualquer faixa etária, provavelmente, por ser a única emoção de valência positiva dentre as emoções básicas (Ku et al., 2005). Outras emoções podem ser confundidas mais frequentemente, tais como as expressões de medo e surpresa, principalmente durante a infância
(Gosselin \& Simard, 1994). Isto parece ocorrer porque até determinado momento da formação da expressão facial, as emoções de medo e de surpresa movimentam os mesmos músculos faciais, sendo discriminadas com maior precisão apenas nos níveis mais elevados da intensidade emocional (Hoffmann, Traue, Walter \& Kessler, 2013). Tal fenômeno ocorre também para com as expressões faciais de nojo e raiva (Jack et al., 2014).

Diferentes métodos têm sido utilizados para avaliar a capacidade do reconhecimento de emoções em faces, sendo a tarefa mais comum a de solicitar aos participantes que nomeiem ou identifiquem a emoção em fotografias de expressões faciais (Ekman \& Heider, 2009). Todavia, críticas têm sido feitas com relação ao uso de estímulos estáticos e com emoção intensa pelo fato de apresentarem menor validade ecológica, já que se distanciam das reais condições de interação social (Chafi, 2012; Fasel \& Luettin, 2003; Goeleven et al., 2008; Roark, Barrett, Spence, Abdi \& O’Toole, 2003; TorroAlves, Bezerra, Claudino \& Pereira, 2013; Willis, Palermo, McGrillen \& Miller, 2014). Além disso, é possível que os seres humanos se utilizem do movimento facial ou da avaliação dos padrões de alteração facial que ocorrem durante a expressão de uma emoção (Maldonado, Rus-Calafell \& González-Conde, 2014; Russell et al., 2003).

Com o objetivo de aumentar a validade ecológica dos estímulos utilizados nos testes de reconhecimento de emoções faciais, estudos têm aplicado a técnica de morphing para produzir expressões faciais com variações no nível de intensidade emocional (Suzuki, Hoshino, Shigemasu \& Kawamura, 2006; Paiva-Silva, Pontes, Aguiar \& Souza, 2016). Através desse procedimento, podem ser elaborados tanto estímulos faciais estáticos (fotografias), quanto estímulo dinâmicos (vídeos), para a utilização nas tarefas experimentais. 


\section{Reconhecimento de expressões emocionais por crianças}

Desde o primeiro ano de vida, os bebês já são capazes de discriminar entre várias expressões emocionais (Hess, Jr \& Kleck, 2004; Trevarthen, 1984), sendo as capacidades de reconhecimento emocional aperfeiçoadas ao longo do desenvolvimento humano (Freitag \& Schwarzer, 2009; Haan, Belsky, Reid, Volein \& Johnson, 2004; Russell \& Fernández-Dols, 1997).

Lawrence, Campbell e Skuse (2015) verificaram maior progresso no reconhecimento das emoções de alegria, surpresa, medo e nojo ao longo do desenvolvimento infantojuvenil, enquanto que pequenas alterações no reconhecimento das expressões de tristeza e raiva foram observadas no mesmo período. Alguns estudos sugerem não haver diferenças entre sexos na infância diante de tarefas de reconhecimento de emoção faciais (Brody \& Harrison, 1987), embora outros mostrem distinção no desempenho entre meninos e meninas (Maxim \& Nowicki, 2003; McClure, 2008).

Durante a infância, o reconhecimento de expressões faciais pode ser influenciado por diferentes fatores como inteligência, puberdade, nível socioeconômico e transtornos comportamentais (Blakemore, 2008; Lawrence et al., 2015). Por exemplo, Machado et al. (2008) verificaram que crianças que possuem maior conhecimento das emoções tendem a responder de maneira mais assertiva às solicitações de professores e pares, construindo interações sociais mais ricas, o que repercute positivamente no sucesso escolar, na autoestima da criança e em seu desenvolvimento (Izard et al., 2001).

Pesquisas realizadas com a técnica de morphing sugerem existir variações entre as crianças na capacidade de reconhecer emoções em faces em um contínuo emocional (Brotman, Skup et al., 2008; Brotman, Guyer et al., 2008; Kessels et al., 2014). É possível que crianças que necessitem de maior intensidade emocional para reconhecimento da expressão facial apresentem proporcionalmente mais dificuldade de adaptação social, já que demoram a compreender as solicitações de outras pessoas. Maxim e Nowicki (2003) afirmam que tal dificuldade pode predizer prejuízos na responsividade às demandas sociais na infância.

No presente estudo, foi avaliado o reconhecimento de emoções faciais por crianças entre 7 e 11 anos, utilizando uma tarefa experimental com variação da intensidade emocional através da técnica de morphing. Partiuse de hipótese de que haveria uma melhora gradual do reconhecimento de expressões faciais com o avanço da idade, assim como diferenças com relação ao nível mínimo de intensidade necessária para o reconhecimento das diferentes emoções faciais.

\section{Método}

\section{Participantes}

Participaram da pesquisa 28 crianças entre 7 e 11 anos de idade, de ambos os sexos, estudantes do $1^{\mathrm{O}}$ ao $6^{\mathrm{O}}$ ano do Ensino Fundamental de uma escola privada de Brasília. Houve distribuição igualitária dos participantes por sexo entre as cinco idades, resultando a seguinte disposição: quatro crianças de 7 anos; seis de 8 anos; seis de 9 anos; oito de 10 anos; e quatro participantes de 11 anos. Foram critérios de exclusão: QI inferior ao percentil 25 (avaliado pelo Teste Raven) e indicativos clínicos de presença de transtornos de ansiedade, depressão e transtorno de déficit de atenção e hiperatividade (avaliado pelo CBCL).

\section{Instrumentos}

Foram utilizados os seguintes instrumentos:

- Matrizes Progressivas Coloridas de Raven (Angelini, Alves, Custódio, Duarte \& Duarte, 1999);

- Child Behavior Checklist - CBCL (Bordin, Mari \& Caeiro, 1995);

- Questionário de Rastreio do Desenvolvimento, que buscou dados do histórico médico e do desenvolvimento neuropsicomotor da criança; 
Juliana Silva Rocha Aguiar, Ana Idalina de Paiva Silva, Carla Silva Rocha Aguiar, Nelson Torro-Alves, ET AL.

- Termo de Consentimento Livre e Esclarecido e Termo de Assentimento;

- Notebook Dell Vostro V 14T - 5470 A60 Touchscreen de 14 polegadas - Windows 8, para desenvolver e apresentar o Teste de Reconhecimento de Emoções em Face Infantil (TREFI).

\section{Estímulos e Tarefa}

O Teste de Reconhecimento de Emoções em Face Infantil (TREFI) foi desenvolvido por esta equipe a partir de fotografias retiradas do Banco de Expressões Emocionais Brasileiro (Batista, Rodrigues \& Torro-Alves, 2013), cedidas e autorizadas pelos autores. Foram selecionadas as fotografias que obtiveram maiores índices no reconhecimento das emoções pelos participantes na etapa de validação das imagens (Batista et al., 2013). Foram selecionadas cinco expressões emocionais de cada uma das seis emoções básicas e as faces neutras dos modelos. Por meio da técnica de morphing, com a utilização do programa Morpheus Photo Animation Suite e do Adobe Photoshop, foram criados seis níveis de intensidade para cada emoção $(25 \%, 40 \%, 55 \%$, $70 \%, 85 \%$ e $100 \%)$.

No total, foram criados 28 conjuntos de imagens ( 4 conjuntos para a fase de treino e 24 para a fase de testagem). Cada conjunto era composto por seis fotografias referentes às diferentes intensidades emocionais (com quatro conjuntos de imagens para cada emoção). Isto significa que foi gerado um banco com um total de 168 de imagens manipuladas, das seis emoções básicas, todas apresentadas no formato Imagem JPEG com tamanho de $360 \mathrm{~KB}$.

O TREFI é organizado do seguinte modo: a primeira tela consiste no preenchimento do cadastro com informações demográficas do participante e pontuação do teste Raven. A segunda tela apresenta as instruções do TREFI ("Você vai ver na tela algumas fotos. Olhe com atenção e diga o que cada uma das pessoas está sentindo: pode ser Alegria, Tristeza, Medo, Surpresa, Nojo ou Raiva. Se você ainda não souber o que ela está sentindo, diga 'Não Sei'.
Vamos começar!"). Após a realização da fase de treino, eram apresentados randomicamente os 24 conjuntos de imagens do teste.

Em cada tela havia uma fotografia centralizada e abaixo dela sete opções de resposta: "Alegria", "Tristeza", "Raiva", "Nojo", "Surpresa", "Medo" e "Não Sei", através de um método de escolha semiforçada. Cada conjunto de imagens era apresentado sequencialmente, sempre iniciando na intensidade de $25 \%$, seguida pelas intensidades de 40\%, 55\%, 70\%, 85\% e $100 \%$. Se a criança selecionasse uma das seis opções de emoção, a sequência era interrompida e um novo conjunto de imagens era apresentado. Caso a criança escolhesse a opção "Não Sei", uma foto de maior intensidade emocional do mesmo conjunto era apresentada, de acordo com a sequência $(25 \%, 40 \%, 55 \%, 70 \%, 85 \%$ e $100 \%)$.

\section{Procedimento}

A coleta de dados foi dividida em três etapas, que ocorreram em dias distintos: 1) autorização dos pais/responsáveis através da assinatura do Termo de Consentimento Livre e Esclarecido - TCLE, preenchimento do Questionário de Rastreio do Desenvolvimento e do Child Behavior Checklist - CBCL (Bordin et al., 1995); 2) aplicação individual do teste Matrizes Progressivas Coloridas de Raven (Angelini et al., 1999) e assinatura do Termo de Assentimento pelas crianças autorizadas; 3) aplicação do TREFI.

Participaram da segunda etapa apenas as crianças autorizadas. Após a assinatura do Termo de Assentimento, era aplicado o teste Matrizes Progressivas Coloridas de Raven, com duração média da sessão de 25 minutos. Em momento posterior, as crianças foram convidadas a responder o TREFI no computador com auxílio da pesquisadora, em uma sessão de aproximadamente 3 a 5 minutos de duração por participante. 


\section{Análise dos Dados}

Para a análise descritiva, foram aplicados os testes de Shapiro-Wilk (idade, QI e intensidade emocional), o teste binomial (sexo), o teste de distribuição uniforme (emoção) e o teste VDXWHe Kendall (para avaliar as correlações entre as variáveis supracitadas). Para a análise inferencial, utilizou-se o método de Modelos Lineares Mistos Generalizados (GLMM).

\section{Resultados}

$\mathrm{Na}$ análise de dados, os testes estatísticos indicaram distribuição normal para as variáveis testadas. Para as variáveis Sexo e Emoções, foi comparada a frequência de participantes em cada categoria, encontrando-se grupos balanceados. Ao se avaliar a frequência de acertos por sexo dos participantes, não houve diferença significativa entre meninos e meninas $(z=-0.574$ e $p=0.56)$.

Quando se avaliou as respostas dos participantes diante da emoção apresentada, as maiores taxas de acerto ocorreram para alegria, enquanto um pior desempenho ocorreu para faces de medo, frequentemente confundidas com surpresa. As demais emoções apresentaram uma quantidade de erros e acertos similares (Figura 1). Tais taxas foram significativas, quando comparadas à emoção de alegria, como detalhado a seguir pela análise GLMM (Tabela 2).

\section{Figura 1}

Gráfico de mosaico para a frequência de acertos e erros por tipo de emoção.

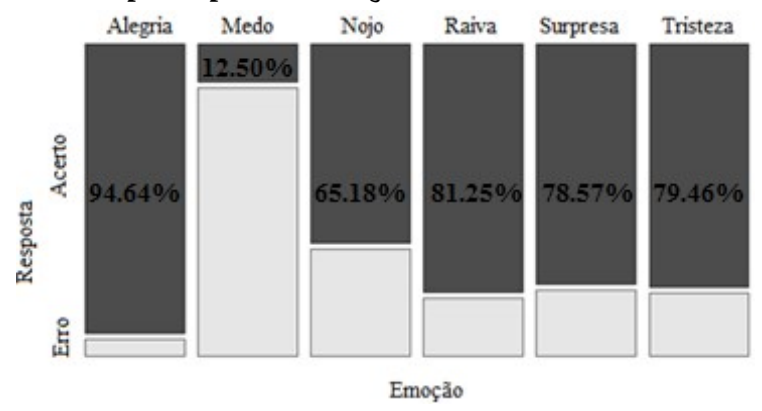

Fonte: elaboração.

Houve uma melhora de desempenho com aumento da idade, visto que a diferença entre a taxa de acertos é menor para crianças de 7 anos, e maior para as de 11 anos (Figura 2). No entanto, pela análise GLMM observamos que

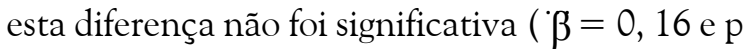
$=0,124$ ), o que será detalhado a seguir (Tabela 2).

\section{Figura 2}

Gráfico de mosaico para a frequência de acertos e erros por idade.

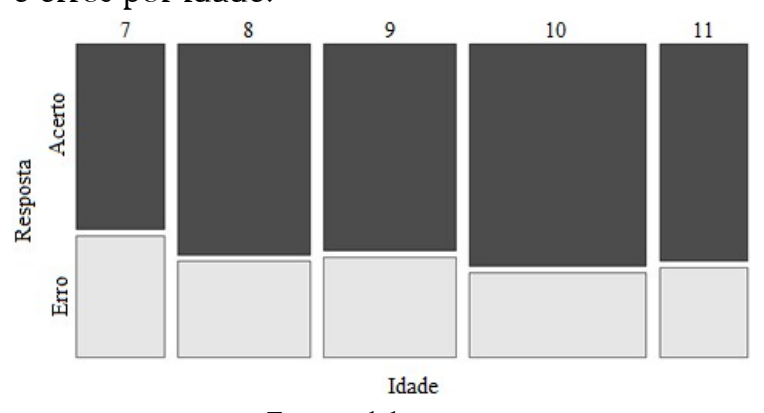

Fonte: elaboração.

Houve uma maior quantidade de erros na baixa intensidade (25\%) para qualquer emoção (Figura 3). Verificou-se que, quanto maior a intensidade emocional da face apresentada, maior foi a diferença entre a quantidade de acertos e erros (Figura 3). A significância da diferença e efeito positivo do aumento da intensidade emocional foi confirmada pela análise GLMM ( $\mathbb{\beta}=0,42$ e p $<0,001)$, o que será detalhado a seguir (Tabela 2).

\section{Figura 3}

Gráfico para a frequência de acertos e erros por intensidade da emoção.

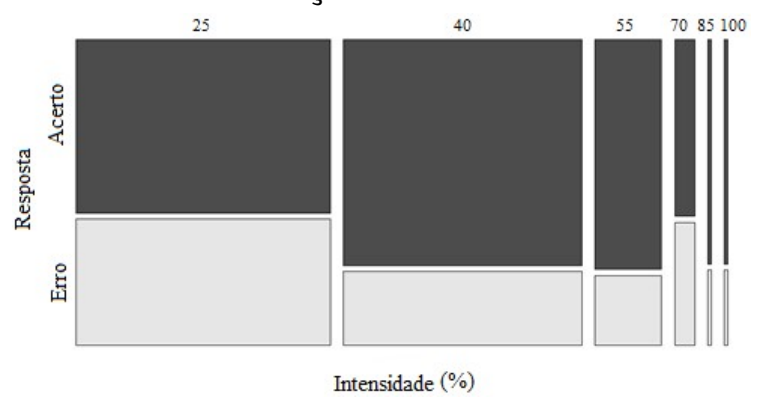

Fonte: elaboração.

Quanto à frequência de intensidade por emoção, houve uma concentração de respostas dadas nas intensidades emocionais de $25 \%$ e $40 \%$, para todas as emoções testadas (Tabela 1). 
Juliana Silva Rocha Aguiar, Ana Idalina de Paiva Silva, Carla Silva Rocha Aguiar, Nelson Torro-Alves, ET AL.

\section{TABELA 1}

Frequência de intensidade e intensidade média por emoção.

\begin{tabular}{lccccccc}
\hline & $25 \%$ & $40 \%$ & $55 \%$ & $70 \%$ & $85 \%$ & $100 \%$ & $\begin{array}{c}\text { Intensidade } \\
\text { Média (DP) }\end{array}$ \\
\hline Alegria & 33 & 45 & 4 & 2 & 0 & 0 & $35.54(8.09)$ \\
Medo & 45 & $51 \mid$ & 8 & 6 & 1 & 1 & $39.91(11.65)$ \\
Nojo & 59 & 35 & 13 & 2 & 1 & 2 & $35.85(10.38)$ \\
Raiva & 48 & 48 & 11 & 5 & 0 & 0 & $36.38(8.57)$ \\
Surpresa & 54 & 37 & 17 & 2 & 1 & 0 & $36.21(10.23)$ \\
Tristeza & 39 & 45 & 20 & 5 & 1 & 1 & $37.59(11.56)$ \\
\hline
\end{tabular}

Fonte: elaboração.

Pela análise inferencial, utilizando Modelos Lineares Mistos Generalizados (GLMM), a variável "intensidade" foi considerada intervalar pois os participantes não passaram pelas mesmas intensidades a cada emoção. Foram aplicadas medidas padronizadas das variáveis preditoras contínuas (idade e intensidade) e a variável de referência para a variável preditora categórica (emoção) foi a expressão de alegria. Assim, o intercepto do modelo representa uma pessoa de 9,11 anos, julgando uma expressão facial de alegria com intensidade de 36, 9\%.

Dos resultados gerais dos efeitos fixos do GLMM (Tabela 2), foi verificado que a idade apresenta um efeito positivo, mas não significativo, sobre a probabilidade de acerto da expressão facial $(\beta=0.16$ e $p=0.124)$. A intensidade emocional, por outro lado, apresentou um efeito positivo e significativo ( $\beta$ $=0,42$ e $p<0.001)$. Por fim, identificou-se que todas as emoções são menos prováveis de se acertar do que a emoção de alegria $(\beta \mathrm{s}<$ $-1,2$, ps $<0.05)$, sendo a emoção de medo a mais distinta $(\beta=-4.7372$ e $p<0.001)$. Já o aumento de uma unidade da intensidade aumenta significativamente a chance de acerto, em $42 \% \quad(\beta=0.4230$ e $<0.001)$; logo, quanto maior a intensidade emocional, maior a probabilidade de acerto.

\section{TABELA 2}

Estimativas dos efeitos fixos, a partir da análise do GLMM

\begin{tabular}{lcccccc}
\hline & $\beta$ & $\mathrm{OR}$ & $95 \% \mathrm{IC}$ & $\mathrm{EP}$ & $z$ & $p$ \\
\hline $\begin{array}{l}\text { Intercepto } \\
\text { (Alegria) }\end{array}$ & 2.668 & 14.412 & {$[6.21-33.43]$} & 0.429 & 6.214 & $<0.001^{* * *}$ \\
Idade & 0.1657 & 1.180 & {$[0.95-1.45]$} & 0.107 & 1.536 & 0.124 \\
Intensidade & 0.4230 & 1.526 & {$[1.21-1.92]$} & 0.117 & 3.594 & $<0.001^{* * *}$ \\
Medo & -4.7372 & 0.008 & {$[0.003-0.024]$} & 0.528 & -8.966 & $<0.001^{* * *}$ \\
Nojo & -1.9625 & 0.140 & {$[0.05-0.35]$} & 0.472 & -4.152 & $<0.001^{* * *}$ \\
Raiva & -1.1258 & 0.324 & {$[0.12-0.85]$} & 0.491 & -2.291 & $0.022^{*}$ \\
Surpresa & -1.2384 & 0.289 & {$[0.11-0.75]$} & 0.487 & -2.538 & $0.011^{*}$ \\
Tristeza & -1.2888 & 0.275 & {$[0.10-0.72]$} & 0.490 & -2.628 & $0.008^{* *}$ \\
\hline
\end{tabular}

Nota: ${ }^{*} p<0.05, * * p<0.01, * * * p<0.001$; onde não marcado, $p>0.05$

Fonte: elaboração.

\section{Discussão}

Os resultados indicaram o aumento da idade como uma tendência de maior probabilidade de acerto no julgamento da emoção avaliada, mas tal relação não foi significativa. Este dado corrobora as hipóteses iniciais deste trabalho e achados científicos prévios (Freitag \& Schwarzer, 2009; Leitzke \& Pollak, 2016). O sexo dos participantes também não foi preditor de mais acertos, visto que crianças de ambos os sexos tiveram desempenho similar no TREFI.

Taxas mais altas de acerto foram encontradas para a emoção de alegria, enquanto um pior desempenho ocorreu para faces de medo. Quando comparadas, identificou-se que todas as emoções são menos prováveis de se acertar do que a alegria. Tais achados estão de acordo com a literatura, sugerindo que a alegria é facilitada por ser a única emoção de valência positiva e, portanto, mais distinta (Ku et al., 2005; Rodger et al., 2015).

Medo foi a expressão emocional que as crianças foram menos capazes de reconhecer. Tal padrão foi encontrado também em outros estudos (Rees et al., 2014; Uljarevic \& Hamilton, 2013). Entretanto, ainda que outras pesquisas tenham achado resultados similares, o resultado parece incoerente, dada a importância do medo para a sobrevivência da espécie (LeDoux, 2003). Shahrestani, Kemp e Guastella (2013) ressaltam que o medo e a alegria são as emoções que mais promovem a adaptabilidade social ao serem 
bem reconhecidas. Neste ponto, é provável que o nível de intensidade emocional usado no presente trabalho tenha interferido no resultado, visto que o medo é constantemente confundido com surpresa em baixas intensidades emocionais (Hossain, Muhammad, Alhamid, Song \& AlMutib, 2016; Jack et al., 2014; Stanley, Zhang, Fung \& Isaacowitz, 2013), assim como o ocorrido na presente pesquisa.

Houve concentração de respostas nas intensidades emocionais de $25 \%$ e $40 \%$ para todas as emoções testadas. Contudo, uma maior quantidade de erros também ocorreu na intensidade de $25 \%$ da emoção, sugerindo que baixas intensidades emocionais são mais difíceis de serem identificadas. Assim, quanto maior a intensidade emocional da face apresentada, melhor o desempenho dos participantes. Este efeito foi significativo, pois a cada incremento na intensidade emocional, aumentou-se a chance de acerto em $42 \%$. Este foi o principal resultado da presente pesquisa, pois vai ao encontro da hipótese de quanto maior a intensidade emocional, maior a probabilidade de reconhecer uma emoção facial (Recio et al., 2014). Portanto, um instrumento que apresente diferentes níveis de intensidade da emoção deve ser mais sensível para detectar as diferenças entre os indivíduos e, possivelmente, mais ecológico (Paiva-Silva et al., 2016; Sato et al., 2013).

Com base nesta discussão, é importante que futuros estudos continuem a avaliar o efeito da variação de intensidade emocional no reconhecimento de emoção em faces, uma vez que os resultados para esta relação foram expressivos. Tais resultados indicaram que $\mathrm{o}$ tipo de metodologia escolhida para avaliar emoções faciais parece interferir nos tipos de emoção avaliados. Novos estudos com amostras maiores, incluindo várias etapas do desenvolvimento humano, podem possibilitar maior uma generalização dos achados.

\section{Referências}

Angelini, A. L., Alves, I. C. B., Custódio, E. M., Duarte, W. F., \& Duarte, J. L. M. (1999).
Manual matrizes progressivas coloridas de Raven: escala especial. Centro Editor de Testes e Pesquisas em Psicologia. São Paulo.

Batista, N. S., Rodrigues, M. R., \& Torro-Alves, N. (2013). Composição e validação de um banco de expressões faciais brasileiro. In XII Congresso Brasileiro de Neuropsicologia e IV Reunião Anual do IBNEC. São Paulo.

Blakemore, S. J. (2008). The social brain in adolescence. Nature Reviews Neuroscience, 9(4), 267-277.

Bordin, I. A. S., Mari, J. J., \& Caeiro, M. F. (1995). Validation of the brazilian version of the child behavior checklist (CBCL): preliminary data. Revista ABP-APAL, 17, $55-66$.

Brody, L. R. \& Harrison, R. H. (1987). Developmental changes in children's abilities to match and label emotionally laden situations. Motivation and Emotion, 11(4), 347-365.

Brotman, M. A., Guyer, A. E., Lawson, E. S., Horsey, S. E., Rich, B. A., ... Leibenluft, E. (2008). Facial emotion labeling deficits in children and adolescents at risk for bipolar disorder. The American Journal of Psychiatry, 165, 385-389.

Brotman, M. A., Skup, M., Rich, B. A., Blair, K. S., Pine, D. S., ... Leibenluft, E. (2008). Risk for bipolar disorder is associated with face processing deficits across emotions. Journal of the American Academy of Child and Adolescent Psychiatry, 47(12), 1455-1461.

Bruce, V. \& Young, A. (1986). Understanding face recognition. British Journal of Psychology, 77(3), 305-327.

Chafi, A. (2012). Three patterns of motion which change the perception of emotional faces. Psychology, 3(1), 82-89.

Darwin, C. (1872/2004). A expressão das emoções no homem e nos animais (C. das Letras, Ed.). São Paulo.

Dhall, A., Goecke, R., Joshi, J., Wagner, M., \& Gedeon, T. (2013). Emotion recognition in the wild challenge 2013. In Proceedings of the 15 th acm on international conference on multimodal interaction (pp. 509-516). ICMI' 13. Sydney, Australia: ACM. 
Juliana Silva Rocha Aguiar, Ana Idalina de Paiva Silva, Carla Silva Rocha Aguiar, Nelson Torro-Alves, ET AL.

Ekman, P. \& Heider, K. G. (2009). The universality of a contempt expression: a replication. Motivation and Emotion, 12(3), 303-308.

Ekman, P. (1992). An argument for basic emotions. Cognition and Emotion, 6(3/4) 169-200.

Fasel, B. \& Luettin, J. (2003). Automatic facial expression analysis: a survey. Pattern Recognition, 36(1), 259-275.

Freitag, C. \& Schwarzer, G. (2009). Influence of emotional facial expressions on 3-5-yearolds' face recognition. Cognitive Development, 26(3), 230-247.

Gauthier, I., Tarr, M. J., Anderson, A. W., Skudlarski, P., \& Gore, J. C. (1999). Activation of the middle fusiform 'face area' increases with expertise in recognizing novel objects. Nature Neuroscience, 2(6), 568-573.

Goeleven, E., Raedt, R. D., Leyman, L., \& Verschuere, B. (2008). The karolinska directed emotional faces: a validation study. Cognition and Emotion, 22 (6), 1094-1118.

Gosselin, P. \& Simard, J. (1994). Children's knowledge of facial expressions of emotions: distinguishing fear and surprise. The Journal of Genetic Psychology, 160(2), 181- 193.

Haan, M., Belsky, J., Reid, V., Volein, A., \& Johnson, M. H. (2004). Maternal personality and infants' neural and visual responsivity to facial expressions of emotion. Journal of Child Psychology and Psychiatry, 45(7), 1209-1218.

Haxby, J. V., Hoffman, E. A., \& Gobbini, M. I. (2000). The distributed human neural system for face perception. Trends in Cognitive Sciences, 4(6), 223-233.

Hess, U., Jr, R. B. A., \& Kleck, R. E. (2004). Facial appearance, gender, and emotion expression. Emotion, 4(4), 378-388.

Hoffmann, H., Traue, H. C., Walter, K. L.-E. S., \& Kessler, H. (2013). Static and dynamic presentation of emotions in different facial areas: fear and surprise show influences of temporal and spatial properties. Psychology, 4(8), 663-668.
Hofmann, S. G., Suvak, M., \& Litz, B. T. (2006). Sex differences in face recognition and influence of facial affect. Personality and Individual Differences, 40(8), 1683-1690.

Hossain, M. S., Muhammad, G., Alhamid, M. F., Song, B., \& Al-Mutib, K. (2016). Audio-visual emotion recognition using big data towards $5 \mathrm{~g}$. Mobile Networks and Applications, 1, 1-11.

Izard, C., Fine, S., Schultz, D., Mostow, A., Ackerman, B., \& Youngstrom, E. (2001). Emotion knowledge as a predictor of social behavior and academic competence in children at risk. Psychological Science, 12(1), 18-23.

Jack, R. E., Garrod, O. G., \& Schyns, P. G. (2014). Dynamic facial expressions of emotion transmit an evolving hierarchy of signals over time. Current Biology, 24(2), 187- 192.

Kawasaki, H., Tsuchiya, N., Kovach, C. K., Nourski, K. V., Oya, H., ... Adolphs, R. (2011). Processing of facial emotion in the human fusiform gyrus. Journal of Cognitive Neuroscience, 24(6), 1358-1370.

Kessels, R. P., Montagne, B., Hendriks, A. W., Perrett, D. I., \& Haan, E. H. (2014). Assessment of perception of morphed facial expressions using the emotion recognition task: normative data from healthy participants aged 8-75. Journal of Neuropsychology, 8(1), 75-93.

Ku, J., Jang, H. J., Kim, K., Kim, J.-H., Park, S. H., ... Kim, S. I. (2005). Experimental results of affective valence and arousal to avatar's facial expressions. Cyberpsychology, Behavior, and Society Networking, 8, 493 503.

Lawrence, K., Campbell, R., \& Skuse, D. (2015). Age, gender, and puberty influence the development of facial emotion recognition. Frontiers in Psychology, 16. https://doi.org/10 $.3389 /$ fpsyg.2015.00761

LeDoux, J. (2003). The emotional brain, fear, and the amygdala. Cellular and Molecular Neurobiology, 23(4-5), 727-738.

Leitzke, B. T. \& Pollak, S. D. (2016). Developmental changes in the primacy 
of facial cues for emotion recognition. Developmental Psychology, 52(4), 572-581.

Machado, P., Veríssimo, M., Torres, N., Peceguina, I., Santos, A. J., \& Rolão, T. (2008). Relações entre o conhecimento das emoções, as competências acadêmicas, as competências sociais e a aceitação entre pares. Análise Psicológica, 3(26), 463-478.

Maldonado, J. G., Rus-Calafell, M., \& GonzálezConde, J. (2014). Creation of a new set of dynamic virtual reality faces for the assessment and training of facial emotion recognition ability. Virtual Reality, 18(1), 61-71.

Maxim, L. A. \& Nowicki, S. J. (2003). Developmental associations between nonverbal ability and social competence. Facta Universitatis-Series Philosophy, Sociology, Psychology and History, 10, 745-758.

McClure, E. B. (2008). A meta-analytic review of sex differences in facial expression processing and their development in infants, children, and adolescents. Psychological Bulletin, 3(126), 424-453.

Meletti, S. (2016). Emotion recognition. Neuropsychiatric Symptoms of Epilepsy, 177193.

Murtaza, M., Sharif, M., Raza, M., \& Shah, J. H. (2013). Analysis of face recognition under varying facial expression: a survey. International Arab Journal of Information Technology (IAJIT), 10(4), 378-388.

Paiva-Silva, A. I., Pontes, M. K., Aguiar, J. S. R., $\&$ Souza, W. C. (2016). How do we evaluate facial emotion recognition? Psychology $\mathbb{E}$ Neuroscience, 9(2), 153-175.

Recio, G., Schacht, A., \& Sommer, W. (2014). Recognizing dynamic facial expressions of emotion: specificity and intensity effects in event-related brain potentials. Biological Psychology, 96, 111-125.

Rees, E. M., Farmer, R., Cole, J. H., Henley, S. M., Sprengelmeyer, R., ... Tabrizi, S. J. (2014). Inconsistent emotion recognition deficits across stimulus modalities in huntington's disease. Neuropsychologia, 64, 99-104.
Roark, D. A., Barrett, S. E., Spence, M. J., Abdi, H., \& O'Toole, A. J. (2003). Psychological and neural perspectives on the role of motion in face recognition. Behavioral and Cognitive Neuroscience Reviews, 2(1), 1546.

Rodger, H., Vizioli, L., Ouyang, X., \& Caldara, R. (2015). Mapping the development of facial expression recognition. Developmental Science, 18(6), 926-939.

Rodrigues, H. \& Rocha, F. L. (2016). Uma definição constitutiva de emoções: a constitutive definition of emotions. Revista Húmus, 5(15), 18-32.

Russell, J. A., Bachorowski, J. A., \& FernandezDols, J. M. (2003). Facial and vocal expressions of emotion. Annual Review of Psychology, 54(1), 329-349.

Russell, J. A. \& Fernández-Dols, J. M. (1997). A what does a facial expression mean? The Psychology of Facial Expression, 1, 10-30.

Sato, W., Uono, S., \& Toichi, M. (2013). A typical recognition of dynamic changes in facial expressions in autism spectrum disorders. Research in Autism Spectrum Disorders, 7(7), 906-912.

Shahrestani, S., Kemp, A. H., \& Guastella, A. J. (2013). The impact of a single administration of intranasal oxytocin on the recognition of basic emotions in humans: a meta-analysis. Neuropsychopharmacology, 38(10), 19291936.

Siegman, A. W. \& Feldstein, S. (2014). Nonverbal behavior and communication (Second edition). New York: Ed Press.

Stanley, J. T., Zhang, X., Fung, H., \& Isaacowitz, D. M. (2013). Cultural differences in gaze and emotion recognition: americans contrast more than chinese. Emotion, 13(1), 36.

Suzuki, A., Hoshino, T., Shigemasu, K., \& Kawamura, M. (2006). Disgust-specific impairment of facial expression recognition in parkinson's disease. Brain, 129(3), 707717.

Tanaka, A., Akamatsu, N., Yamano, M., Nakagawa, M., Kawamura, M., \& Tsuji, S. 
Juliana Silva Rocha Aguiar, Ana Idalina de Paiva Silva, Carla Silva Rocha Aguiar, Nelson Torro-Alves, ET AL.

(2012). A more realistic approach, using dynamic stimuli, to test facial emotion recognition impairment in temporal lobe epilepsy. Psychometrika, 38(1), 116-133.

Torro-Alves, N., Bezerra, I. A. O., Claudino, R. G., \& Pereira, T. C. L. (2013). Influences of sex, type and intensity of emotion in the recognition of static and dynamic facial expressions. Avances en Psicología Latinoamericana, 31, 192-199.

Trevarthen, C. (1984). Facial expressions of emotion in mother-infant interaction. human neurobiology. Attachment $\& 3$ Human Development, 4(1), 21-32.

Uljarevic, M. \& Hamilton, A. (2013). Recognition of emotions in autism: a formal metaanalysis. Journal of Autism and Developmental Disorders, 43 (7), 1517-1526.

Weiner, K. S. \& Zilles, K. (2015). The anatomical and functional specialization of the fusiform gyrus. Neuropsychologia, 83, 48-62.

Willis, M. L., Palermo, R., McGrillen, K., \& Miller, L. (2014). The nature of facial expression recognition deficits following orbitofrontal cortex damage. Neuropsychology, 28(4), 613-623.

\section{Notas}

* Research Article. 